\title{
Investigation of Base-free Copper-Catalysed Azide-Alkyne Click Cycloadditions (CuAAc) in Natural Deep Eutectic Solvents as Green and Catalytic Reaction Media
}

\author{
Salvatore V. Giofrè,${ }^{1 *}$ Matteo Tiecco, ${ }^{2 *}$ Angelo Ferlazzo, ${ }^{3}$ Roberto Romeo, ${ }^{1}$ Gianluca \\ Ciancaleoni, ${ }^{4}$ Raimondo Germani ${ }^{2}$ and Daniela Iannazzo ${ }^{3}$
}

1. Dipartimento di Scienze Chimiche, Biologiche, Farmaceutiche ed Ambientali, Università di Messina, Viale Annunziata, I-98168 Messina, Italy.

2. Dipartimento di Chimica, Biologia e Biotecnologie, Università di Perugia, via Elce di Sotto 8, I06123 Perugia, Italy.

3. Dipartimento di Ingegneria, Università of Messina, Contrada Di Dio, I-98166 Messina, Italy

4. Dipartimento di Chimica e Chimica Industriale (DCCI), Università di Pisa, Via Giuseppe Moruzzi, 13, I-56124 Pisa, Italy.

* Corresponding authors

Email addresses: sgiofre@unime.it (Salvatore V. Giofrè); matteotiecco@gmail.com (Matteo Tiecco).

\begin{abstract}
The click cycloaddition reaction of azides and alkynes affording 1,2,3-triazoles is a transformation widely used to obtain relevant products in chemical biology, medicinal chemistry, materials science and other fields. In this work, a set of Natural Deep Eutectic Solvents (NADESs) as "active" reaction media has been investigated in the copper-catalysed azide-alkyne cycloaddition reactions (CuAAc). The use of these green liquids as green and catalytic solvents has shown to improve the reaction effectiveness, giving excellent yields. The NADESs proved to be "active" in this transformation for the absence of added bases in all the performed reactions and in several cases for their reducing capabilities. The results were rationalized by DFT calculations which demonstrated the involvement of H-bonds between DESs and alkynes as well as a stabilization of copper catalytic intermediates. The green experimental conditions, namely the absence of a base, the low temperatures, the lowering
\end{abstract}


of reagents and the possibility of recycling of these green solvents, outline the great potential of NADESs for CuAAc and in general, for green organic synthesis.

\section{KEYWORDS}

Click Chemistry; Copper-catalysed Azide-Alkyne Cycloadditions; Natural Deep Eutectic Solvents; Catalytic Green Solvents; Recycling of Green Solvents; DFT Calculations.

\section{INTRODUCTION}

The development of sustainable chemical processes is of prominent importance in response to the increased awareness and concern over the environmental impact of chemical pollutants. The minimizing of environmental impact of solvents in chemicals production still represents a global challenge for their impact on health cost and safety. ${ }^{1-3}$ The solvent represents the main and most abundant component in a chemical transformation; thus, the substitution of a polluting, toxic and volatile organic solvent with a reaction media that could possess green advantages is probably the most important option to be considered in order to perform a green and environmental-friendly chemical process. ${ }^{4,5}$ In this topic many relevant solutions are emerging from the recent literature to face up to the problem of volatile polluting solvents. ${ }^{6}$ Ionic Liquids (ILs) played a relevant part thanks to their non-volatility and to their recycling capabilities. ${ }^{7-9}$

However, ILs revealed to be toxic and easily accumulating on the environment because of their noneasy biodegradability. ${ }^{10,11}$ Deep Eutectic Solvents (DESs), born as a side-class of ILs, are emerging from the recent literature and they can overcome these matters. DESs are an innovative class of organic liquids at room temperature that possess green and catalytic properties. Recently, these solvents have found fruitful applications in many different topics, such as chemical transformations, extraction/preconcentration procedures, nanoparticle synthesis, $\mathrm{CO}_{2}$ capture, and all the other applications where they are successfully substituted to commonly used polluting solvents, because of their favourable properties. ${ }^{12-16}$ DESs can be divided in many sub-classes depending on the molecules forming them. The great number of molecules available for their formation lead to a high number of liquids available; ${ }^{17-19}$ however, they can be easily described as mixtures of a Hydrogen Bond Donor (HBD) and a Hydrogen Bond Acceptor (HBA) compounds. ${ }^{20}$ The weak interactions occurring between the counterparts lead to a high decrease of the melting points of the mixtures (eutectic point at a determined molar ratio) therefore leading to a liquid system formation. In particular, the different interactions occurring between the same species (HBD-HBD and HBA-HBA) and between the 
different species (HBD-HBA) lead to a difficult lattice regular organization and therefore to liquid mixtures; this "chaotic" structuration leads to systems with unique properties. ${ }^{21}$ Structural studies of these liquids are abundantly reported in literature since the physical and microscopical properties of these liquids, deeply affect their uses in chemical transformations. ${ }^{22,23}$

DESs possess many green properties: they are non-volatile, easily biodegradable, biocompatible, nontoxic; moreover, they can be easily recovered and recycled and can be formed by cheap components. $^{24-26}$

A relevant sub-class of DESs is represented by the NADESs (NAtural DESs): liquids formed by natural-source molecules, therefore mixtures with increased bioavailability, biodegradability and therefore biocompatibility. ${ }^{27,28}$ Many recent and relevant papers report NADESs as solvents used by some living plants in extremely hot or cold conditions to substitute water for the cellular metabolism processes because these liquids possess a larger liquid window compared to water and can be useful in extreme conditions in these biological systems. ${ }^{29-31}$

One of the most important green aspect or property DESs possess relies on their preparation that can be made by simply mixing and heating the HBD and the HBA that are often solids. Therefore, no solvent and no other reactant is needed at all for their preparation that has a $100 \%$ yield and $100 \%$ atom economy. This increase their greenness over other green liquids in a step that is also prior on their use and on the evaluation of the greenness of a chemical transformation. ${ }^{32}$

Over the green properties, DESs can possess catalytic properties. Recent papers report about acid catalytic behaviour, reducing capabilities and also organocatalytic properties of the reaction media itself. $^{33-37}$ This is because the properties of the liquids reflect on the properties of the molecules forming them; moreover, since the molecules that can form a DES are available in great number, the properties of the liquids are tuneable.

Structural studies that emerged from the needing of interpretation of the observed data, shed light on the association constant (or intimacy of HBD-HBA couple) in order to explain the catalytic behaviour of these liquids since the availability of one of the two components is dependent on the "freedom" of it from the counterpart, and this has an impact also with small differences for example on the pKa value of acids that showed different behaviours when mixed in DESs' structures. ${ }^{38-41}$

In this work is reported the use of a set of differently structured NADESs as "active" reaction media in copper-catalysed azide-alkyne cycloaddition reactions (CuAAc).

Since being first introduced by Sharpless ${ }^{42,43}$ and Meldal, ${ }^{44}$ click chemistry has become a privileged reaction in the chemists' toolbox ${ }^{45}$ and it has shown numerous advantages including easy product recovery, inoffensive by-product formation, modular reaction with wide scope and many inexpensive, readily available starting materials, catalyst, ligands, and so on. ${ }^{46}$ Moreover, 1,2,3-triazoles have 
found application to a wide variety of research areas, including organic and pharmaceutical chemistry, bioconjugation, drug discovery, polymer, nano- and material sciences. ${ }^{45-50}$

The different NADESs were chosen to investigate their catalytic behaviour in these chemical reactions. Their reducing abilities, due to their components, is here demonstrated for several of the investigated reactions $(50 \%)$, where no reagents have been needed to reduce $\mathrm{CuSO}_{4}$. Moreover, in none of the experiments a base (such as triethylamine) was added, as DESs proved to weaken the C$\mathrm{H}$ alkyne bond through their H-bonds network, promoting its cleavage, and stabilize copper catalytic intermediates, as demonstrated via DFT studies. The greenness of the NADESs, their catalytic reducing behaviour, the lowering of the number of reagents used, the recycle capabilities of the media and (last but not least) the excellent yields observed, promote this protocol as an efficient green route for this relevant and widely used chemical reaction.

\section{RESULTS AND DISCUSSION}

\section{Selection and properties of the NADESs for the reaction}

In order to fully explore the role of DES in CuAAc reactions, a set of differently structured NADESs was chosen (Figure 1), and each one for different reasons: urea/choline chloride mixture (U/ChCl, molar ratio $2 / 1$, m.p. $=12^{\circ} \mathrm{C}$ ) was used as it represents the most common and widely used DESs for many synthetic applications, ${ }^{51}$ L-ascorbic acid/choline chloride $(\mathbf{A s c} / \mathbf{C h C l}$, molar ratio 1/2, m.p. $=$ $20^{\circ} \mathrm{C}$ ) was selected as the acid component is endowed with reducing capabilities and therefore no ascorbate is needed for the click reaction; ${ }^{52}$ oxalic acid/choline chloride $(\mathbf{O x} / \mathbf{C h C l}$, molar ratio 1/2, m.p. $=30^{\circ} \mathrm{C}$ ) was chosen as it is constituted by another reducing agent, $\mathbf{O x}$, and by the same HBA of Asc/ChCl ${ }^{53}$ oxalic acid/trimethylglycine (Ox/TMG, molar ratio 2/1, m.p. $\left.=33^{\circ} \mathrm{C}\right)$ was used to explore the role of the HBD counterpart of the former liquid and the role of TMG as HBA; ${ }^{53}$ glycolic acid/trimethylglycine $\left(\mathbf{G A} / \mathbf{T M G}\right.$, molar ratio 2/1, m.p. $\left.=-36^{\circ} \mathrm{C}\right)$ was investigated as it represents a model mixture of the zwitterionic DESs for its favourable physical properties and because it is constituted by the same HBA of other two mixtures in our set. ${ }^{54,55}$ 


$$
\mathrm{H}_{\text {urea }} \stackrel{\mathrm{O}}{\stackrel{\mathrm{N}}{\mathrm{NH}_{2}}}
$$<smiles>O=C1OC(CCO)C(O)=C1O</smiles>

ŌH

L-ascorbic acid

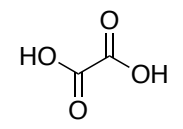

oxalic acid<smiles>O=C(O)C(=O)O</smiles>

oxalic acid

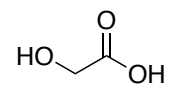

glycolic acid

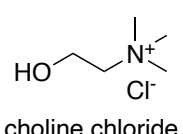

$\mathrm{U} / \mathrm{ChCl}$

choline chloride<smiles>C[N+](C)(C)CCO</smiles>

choline chloride

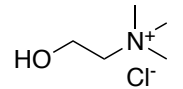

choline chloride<smiles>C[N+](C)(C)CC(=O)O</smiles>

trimethylglycine

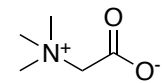

trimethylglycine

$\mathrm{Ox} / \mathrm{ChCl}$

OX/TMG

GA/TMG
$2 / 1$

$12^{\circ} \mathrm{C}$

Asc/ChCl

$1 / 2$

$20^{\circ} \mathrm{C}$

$1 / 2$

$30^{\circ} \mathrm{C}$

2/1

$33^{\circ} \mathrm{C}$

$2 / 1$

$-36^{\circ} \mathrm{C}$

Figure 1. HBD and HBA components, Acronyms, composition and melting points of Deep Eutectic Solvents used in this work.

\section{Chemistry}

To start this work, the 1,3-dipolar cycloaddition of terminal alkynes with organic azides were investigated, using as a model reaction, the classical cycloaddition of benzyl azide and phenylacetylene. The chemical role of DESs components in the cycloaddition processes was investigated under different experimental conditions (temperature, time, in presence or absence of sacrificial reductants). For all the investigated reactions, no base was needed for the reaction effectiveness, as also reported in other studies, ${ }^{56}$ thus confirming the green contribution of DESs in $\mathrm{CuAAc}$ reactions where a base as co-catalyst is required (see theoretical calculations). Moreover, all the reactions were performed at low temperatures, always below $50^{\circ} \mathrm{C}$, so further highlighting the 
advantages introduced by these green solvents. The final products were obtained in most case after addition of water to the crude reaction mixture that allows easy precipitation of the desired triazole without the use of conventional hazardous volatile organic solvents (VOCs) (Table 1).

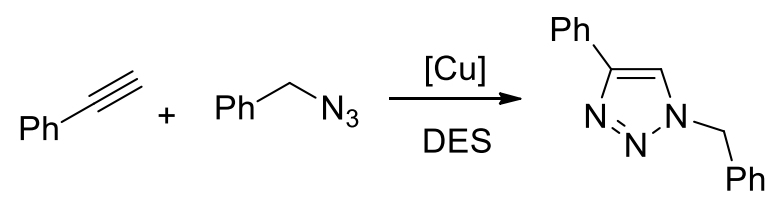

Scheme 1. Copper-catalysed azide-alkyne cycloadditions (CuAAC).

Table 1. Study of the 1,3-dipolar cycloaddition of benzyl azide and phenylacetylene catalysed by the copper salts in different natural deep eutectic solvents. ${ }^{a}$

\begin{tabular}{lllllll}
\hline Entry & Catalyst $\mathrm{Mol}_{0} \%$ & Solvent & Reductant Mol \% & Temperature & Time $[\mathrm{h}]$ & Yield $^{b}$ \\
\hline 1 & $10, \mathrm{CuSO}_{4}$ & GA/TMG & - & r.t. & 72 & - \\
2 & $10, \mathrm{CuSO}_{4}$ & $\mathbf{G A} / \mathbf{T M G}$ & 30 & r.t. & 72 & 99 \\
3 & $10, \mathrm{CuSO}_{4}$ & $\mathbf{G A} / \mathbf{T M G}$ & 30 & r.t. & 12 & 99 \\
4 & $10, \mathrm{CuSO}_{4}$ & $\mathbf{G A} / \mathbf{T M G}$ & 30 & $50{ }^{\circ} \mathrm{C}$ & 3 & 99 \\
5 & $10, \mathrm{CuSO}_{4}$ & $\mathbf{O x} / \mathbf{C h C l}$ & - & $40{ }^{\circ} \mathrm{C}$ & 72 & $95^{c}$ \\
6 & $10, \mathrm{CuSO}_{4}$ & $\mathbf{O x} / \mathbf{C h C l}$ & 30 & $40{ }^{\circ} \mathrm{C}$ & 72 & $95^{c}$ \\
7 & - & $\mathbf{O x} / \mathbf{C h C l}$ & & $40{ }^{\circ} \mathrm{C}$ & 72 & $95^{c}$ \\
8 & $10, \mathrm{CuSO}_{4}$ & $\mathbf{O x} / \mathbf{T M G}$ & - & $50{ }^{\circ} \mathrm{C}$ & 72 & $95^{d}$ \\
9 & $10, \mathrm{CuSO}_{4}$ & $\mathbf{O x} / \mathbf{T M G}$ & 30 & $50{ }^{\circ} \mathrm{C}$ & 72 & $95^{d}$ \\
10 & - & $\mathbf{O x} / \mathbf{T M G}$ & & $50{ }^{\circ} \mathrm{C}$ & 72 & $95^{d}$ \\
11 & $10, \mathrm{CuSO}_{4}$ & $\mathbf{U} / \mathbf{C h C l}$ & - & r.t. & 72 & $57^{e}$ \\
12 & $10, \mathrm{CuSO}_{4}$ & $\mathbf{U} / \mathbf{C h C l}$ & 30 & r.t. & 72 & 96 \\
13 & $10, \mathrm{CuSO}_{4}$ & $\mathbf{U} / \mathbf{C h C l}$ & 30 & r.t. & 24 & 70 \\
14 & $10, \mathrm{CuSO}_{4}$ & $\mathbf{A s c} / \mathbf{C h C l}$ & - & r.t. & 72 & 97 \\
15 & $10, \mathrm{CuSO}_{4}$ & $\mathbf{A s c} / \mathbf{C h C l}$ & - & r.t. & 24 & 97 \\
16 & $10, \mathrm{CuI}$ & $\mathbf{G A} / \mathbf{T M G}$ & - & r.t. & 72 & 35
\end{tabular}

${ }^{a}$ Reactions were performed using $2 \mathrm{~g}$ of the corresponding solvent and $1 \mathrm{mmol}$ of both reagents. ${ }^{b}$ Yields were calculated by ${ }^{1} \mathrm{H}$ NMR. ${ }^{c} 1: 1.2$ ratio (mixture of 1,4- and 1,5-disubstituted 1,2,3-triazole regioisomers). ${ }^{d} 1: 1.5$ ratio (mixture of 1,4and 1,5-disubstituted 1,2,3-triazole regioisomers). ${ }^{e}$ Phenylethynylcopper (I) ladderane polymer as catalyst.

The initial screening for NADESs was done using GA/TMG. As expected, without sodium ascorbate the in situ reduction of $\mathrm{CuSO}_{4} \cdot 5 \mathrm{H}_{2} \mathrm{O}$ did not occur; therefore, after $72 \mathrm{~h}$ only the reagents were found in the reaction mixture (entry 1, Table 1). Conversely, the addition of sodium ascorbate releasing the catalytically reactive $\mathrm{Cu}(\mathrm{I})$ ions, led to the 1,4-disubstituted regioisomer with a 99\% yield in $12 \mathrm{~h}$, in agreement with the results expected from Sharpless reaction ${ }^{42}$ (entry 3, Table 1). The optimized reaction at $50^{\circ} \mathrm{C}$ in $3 \mathrm{~h}$ led to the 1,4-disubstituted regioisomer with a $99 \%$ yield (entry 4, Table 1). It is important to note that the increasing of reaction temperature above $50^{\circ} \mathrm{C}$ led to the browning of the 
reaction mixture due to the degradation of sodium ascorbate. Then, to compare the obtained results, other two DESs were analyzed, $\mathbf{O x} / \mathbf{C h C l}$ and $\mathbf{O x} / \mathbf{T M G}$, based on oxalic acid as HBD, which could act as a reducing agent..$^{57}$ Due to their melting point $\left(30^{\circ} \mathrm{C}\right.$ for $\mathbf{O x} / \mathbf{C h C l}$ and $33^{\circ} \mathrm{C}$ for $\left.\mathbf{O x} / \mathbf{T M G}\right)$, the two green solvents were not used at room temperature and to obtain a homogeneous reaction mixture the experiments were carried out at $40^{\circ} \mathrm{C}$ and $50^{\circ} \mathrm{C}$ respectively. Unexpectedly, the reaction carried out in DES Ox/ $\mathbf{C h C l}$ at $40^{\circ} \mathrm{C}$ for $72 \mathrm{~h}$ led to a mixture of 1,4- and 1,5-disubstituted 1,2,3-triazole regioisomers in 1:1.2 ratio (entry 5 and 6 , Table 1 ), either with and without reducing agents, in according to thermal Huisgen 1,3-dipolar cycloaddition. ${ }^{58,59}$ The same results were obtained using DES Ox/TMG at $50^{\circ} \mathrm{C}$ for $72 \mathrm{~h}$; the reaction performed led to a mixture of 1,4- and 1,5-disubstituted 1,2,3-triazole regioisomers in 1:1.5 ratio (entry 8 and 9, Table 1) either with and without reducing agent. These results can be reasonably attributed to the presence of oxalic acid that acts as a hydrogen bond donor in the formation of DES. Oxalates have been extensively studied as mild reducing agents but also as precipitants and chelating agents. ${ }^{60,61}$ Oxalate ion is a bidentate ligand which forms a fivemembered chelate ring when it binds to metals. In particular, metals such as $\mathrm{Cu}^{2+}$ and $\mathrm{Zn}^{2+}$ can combine with two oxalates, forming a four-coordinate planar square complex. ${ }^{61}$ Based on these considerations, it can be hypothesized that copper is sequestered by DES and the azide-alkyne cycloaddition can follow the Huisgen thermal pathway and not the $\mathrm{Cu}$-catalysed process. It is noteworthy that the classic non-catalysed Huisgen azide-alkyne cycloaddition requires high temperature while in the DESs constituted by oxalic acid, the reaction proceeded under relatively mild conditions at $40-50{ }^{\circ} \mathrm{C}$. These results were also confirmed performing the same reactions without the catalyst $\mathrm{CuSO}_{4}$ and using the same reaction parameters (entries 7 and 10, Table 1); in all the cases, the formation of a mixture of 1,4- and 1,5-disubstituted 1,2,3-triazole regioisomers in the same ratios, was observed.

The DES U/ChCl produced particularly interesting results. To investigate the contribution of this DES in the cycloaddition process, the reaction was carried out using only $\mathrm{CuSO}_{4}$, in the absence of ascorbate, at r.t.. After $72 \mathrm{~h}$, the formation of the 1,4-disubstituted cycloadduct with a 57\% yield was observed (entry 11, Table 1). Surprisingly, the reaction was successful without using the reducing agent, with the formation of a solid yellow insoluble by-product already after $24 \mathrm{~h}$. Therefore, the reaction was further investigated by ${ }^{1} \mathrm{H}$ and ${ }^{13} \mathrm{C}$ NMR. The analysis of the reaction raw material showed the formation of a small amount of 1,4-diphenylbuta-1,3-diyne similarly to what occurs in Glaser reactions. ${ }^{62}$ As reported, a phenylethynylcopper (I) ladderane polymer can catalyse the azidealkyne click chemistry reactions. ${ }^{44}$ Therefore, it was hypothesized that urea actively participated in the reaction process as ligand ${ }^{61}$ by chelating $\mathrm{Cu}(\mathrm{II})$ and subsequently, the copper (I) catalyst, required in the click reactions, resulted from the formation of small amounts of polymeric copper (I) pre- 
catalysts formed in the precursor of Glaser reactions. ${ }^{44}$ Then, the click chemistry reaction in $\mathbf{U} / \mathbf{C h C l}$ solvent was investigated with the presence of the reducing agent and in these conditions, the reaction led to the formation of the 1,4-disubstituted cycloadduct with a $96 \%$ yield. It is noteworthy that in this system we have not observed the formation of the yellow solid, thus assuming that the course of the reaction follows the classical catalytic cycle of the Sharpless reaction (entry 12, Table 1). The reduction of the reaction time led to a decrease in the reaction efficiency (entry 13, Table 1), conversely to what verified with DES GA/TMG (entries 1-4, Table 1).

NADESs based on choline chloride and L-ascorbic acid as green solvent in CuAAC was also investigated. This green solvent can be considered an active DES due to the presence of L-ascorbic acid which could act as a reducing agent in copper-catalysed azide-alkyne cycloadditions. Then, $\mathrm{CuSO}_{4} \cdot 5 \mathrm{H}_{2} \mathrm{O}$ was added to benzyl azide and phenyl acetylene in the base- and reducing agent-free DES solvent and the mixture was left to react for $72 \mathrm{~h}$ at room temperature. As hypothesized, Lascorbic acid produced the copper (I) catalyst required in the click reactions and the 1,4-disubstituted cycloadduct with a $97 \%$ yield was obtained. The decrease in the reaction time was also investigated and a greater efficiency was obtained in $24 \mathrm{~h}$ (entries 14 and 15, Table 1); moreover, a further decrease in reaction times or increase in the reaction temperature, led to loss of yield.

To compare the data reported in literature which show the $\mathrm{Cu}(\mathrm{I})$-catalysed 1,3-dipolar cycloaddition azide-alkyne in different environmentally friendly solvents, ${ }^{56}$ we have investigated the catalytic activity of $\mathrm{CuI}$ in NADES GA/TMG in the base- and reducing agent-free conditions (entry 16, Table 1). The reaction carried out for $72 \mathrm{~h}$ at room temperature afforded the desired 1,4-disubstituted cycloadduct; the observed low yields (35\%) are probably due to the low solubility of the catalyst in the examined DES.

The initial results suggested that NADES GA/TMG represents the potentially best green solvent in our set for the investigated $\mathrm{CuAAc}$ reactions in base-free conditions. Also, the active DES Asc/ChCl showed peculiar characteristics, leading to its use as a green solvent in a non-classical CuAAc baseand reducing agent-free reactions. To extend the scope of this work, our studies were applied to a different azides and terminal alkynes using GA/TMG and Asc/ChCl as green solvents in the optimized experimental conditions described above.

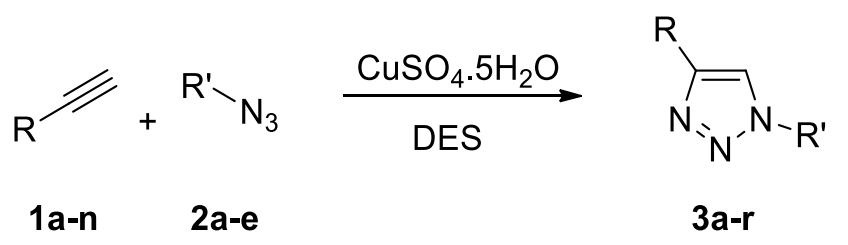

Scheme 2. Copper-catalysed azide-alkyne cycloadditions (CuAAC) in natural deep eutectic solvents. 
Table 2. CuAAc reactions in NADESs ${ }^{*}$.

\begin{tabular}{|c|c|c|c|c|c|c|c|c|c|}
\hline Alkynes & $\mathbf{R}$ & Azides & $\mathbf{R}^{\prime}$ & Solvent & $\begin{array}{l}\text { Red. } \\
\text { Ag. } \\
\text { Mol } \\
\%\end{array}$ & Temp. & $\begin{array}{l}\text { Time } \\
\text { [h] }\end{array}$ & Product & Yield $^{b, c}$ \\
\hline $1 \mathrm{a}$ & $\mathrm{Ph}$ & $2 a$ & $\mathrm{Bn}$ & GA/TMG & 30 & $50^{\circ} \mathrm{C}$ & 3 & $3 a$ & $99(96)$ \\
\hline $1 b$ & $\mathrm{CH}_{2} \mathrm{CH}_{2} \mathrm{OH}$ & $2 a$ & $\mathrm{Bn}$ & GA/TMG & 30 & $50{ }^{\circ} \mathrm{C}$ & 3 & $\mathbf{3 b}$ & $78(65)$ \\
\hline $1 \mathrm{c}$ & $\mathrm{CH}_{2} \mathrm{CH}_{2} \mathrm{CH}_{3}$ & $2 a$ & $\mathrm{Bn}$ & GA/TMG & 30 & $50{ }^{\circ} \mathrm{C}$ & 3 & $3 c$ & $94(90)$ \\
\hline 1d & TMS & $2 a$ & $\mathrm{Bn}$ & GA/TMG & 30 & $50^{\circ} \mathrm{C}$ & 3 & 3d & $95(90)$ \\
\hline $1 e$ & & $2 \mathbf{a}$ & $\mathrm{Bn}$ & GA/TMG & 30 & $50{ }^{\circ} \mathrm{C}$ & 3 & $3 e$ & $95(90)$ \\
\hline 1f & Cyclopropyl & $2 a$ & $\mathrm{Bn}$ & GA/TMG & 30 & $50^{\circ} \mathrm{C}$ & 3 & $3 f$ & $95(92)$ \\
\hline $1 g$ & $t$-butyl & $2 a$ & $\mathrm{Bn}$ & GA/TMG & 30 & $50{ }^{\circ} \mathrm{C}$ & 3 & $3 g$ & $95(92)$ \\
\hline $1 \mathrm{~h}$ & $4-\mathrm{NO}_{2}-\mathrm{Ph}$ & $2 a$ & $\mathrm{Bn}$ & GA/TMG & 30 & $50^{\circ} \mathrm{C}$ & 3 & $3 h$ & $95(90)$ \\
\hline $1 \mathrm{i}$ & 4-pentyl-Ph & $2 a$ & $\mathrm{Bn}$ & GA/TMG & 30 & $50{ }^{\circ} \mathrm{C}$ & 3 & $3 \mathbf{i}$ & $98(95)$ \\
\hline $1 \mathrm{j}$ & 4-MeO-Ph & $2 \mathbf{a}$ & $\mathrm{Bn}$ & GA/TMG & 30 & $50{ }^{\circ} \mathrm{C}$ & 3 & $3 \mathbf{j}$ & $98(94)$ \\
\hline $1 \mathrm{k}$ & 4-F-Ph & $2 a$ & $\mathrm{Bn}$ & GA/TMG & 30 & $50^{\circ} \mathrm{C}$ & 3 & $3 k$ & $98(92)$ \\
\hline 11 & & $2 a$ & $\mathrm{Bn}$ & GA/TMG & 30 & $50^{\circ} \mathrm{C}$ & 3 & 31 & $98(91)$ \\
\hline $1 \mathrm{~m}$ & & $2 a$ & $\mathrm{Bn}$ & GA/TMG & 30 & $50^{\circ} \mathrm{C}$ & 3 & $3 m$ & $98(90)$ \\
\hline $1 n$ & & $2 a$ & $\mathrm{Bn}$ & GA/TMG & 30 & $50^{\circ} \mathrm{C}$ & 3 & $3 n$ & $98(90)$ \\
\hline $1 \mathrm{a}$ & $\mathrm{Ph}$ & $2 b$ & $\mathrm{Ph}$ & GA/TMG & 30 & $50^{\circ} \mathrm{C}$ & 3 & 30 & $95(88)$ \\
\hline $1 \mathrm{a}$ & $\mathrm{Ph}$ & $2 \mathrm{c}$ & & GA/TMG & 30 & $50^{\circ} \mathrm{C}$ & 3 & $3 p$ & $92(86)$ \\
\hline $1 \mathrm{a}$ & $\mathrm{Ph}$ & $2 d$ & $\mathrm{CH}_{2} \mathrm{CH}_{2} \mathrm{OH}$ & GA/TMG & 30 & $50^{\circ} \mathrm{C}$ & 3 & $3 q$ & $97(88)$ \\
\hline $1 \mathrm{a}$ & $\mathrm{Ph}$ & $2 \mathrm{e}$ & 4-MeO-Ph & GA/TMG & 30 & $50^{\circ} \mathrm{C}$ & 3 & $3 r$ & $96(90)$ \\
\hline $1 a$ & $\mathrm{Ph}$ & $2 a$ & $\mathrm{Bn}$ & Asc/ChCl & - & r.t. & 24 & $3 a$ & $97(94)$ \\
\hline $1 b$ & $\mathrm{CH}_{2} \mathrm{CH}_{2} \mathrm{OH}$ & $2 a$ & $\mathrm{Bn}$ & Asc/ChCl & - & r.t. & 24 & $\mathbf{3 b}$ & - \\
\hline $1 \mathrm{c}$ & $\mathrm{CH}_{2} \mathrm{CH}_{2} \mathrm{CH}_{3}$ & $2 a$ & $\mathrm{Bn}$ & Asc/ChCl & - & r.t. & 24 & $3 c$ & - \\
\hline $1 d$ & TMS & $2 a$ & $\mathrm{Bn}$ & Asc/ChCl & - & r.t. & 24 & 3d & $55(48)$ \\
\hline $1 e$ & & $2 a$ & $\mathrm{Bn}$ & Asc/ChCl & - & r.t. & 24 & $3 e$ & - \\
\hline 1f & Cyclopropyl & $2 \mathbf{a}$ & $\mathrm{Bn}$ & $\mathrm{Asc} / \mathrm{ChCl}$ & - & r.t. & 24 & $3 f$ & - \\
\hline $1 g$ & $t$-butyl & $2 a$ & $\mathrm{Bn}$ & Asc/ChCl & - & r.t. & 24 & $3 g$ & - \\
\hline $1 \mathrm{~h}$ & $4-\mathrm{NO}_{2}-\mathrm{Ph}$ & $2 \mathbf{a}$ & $\mathrm{Bn}$ & $\mathrm{Asc} / \mathrm{ChCl}$ & - & r.t. & 24 & $3 \mathrm{~h}$ & $94(88)$ \\
\hline $1 \mathbf{i}$ & 4-pentyl-Ph & $2 a$ & $\mathrm{Bn}$ & Asc/ChCl & - & r.t. & 24 & $3 \mathbf{i}$ & 98(94) \\
\hline $\mathbf{1 j}$ & 4-MeO-Ph & $2 a$ & $\mathrm{Bn}$ & Asc/ChCl & - & r.t. & 24 & $3 \mathbf{j}$ & $87(81)$ \\
\hline $1 \mathrm{k}$ & 4-F-Ph & $2 a$ & $\mathrm{Bn}$ & Asc/ChCl & - & r.t. & 24 & $3 k$ & $88(80)$ \\
\hline
\end{tabular}




\begin{tabular}{|c|c|c|c|c|c|c|c|c|c|}
\hline 11 & & $2 a$ & $\mathrm{Bn}$ & Asc/ChCl & - & r.t. & 24 & 31 & $92(85)$ \\
\hline $1 \mathrm{~m}$ & & $2 a$ & $\mathrm{Bn}$ & Asc/ChCl & - & r.t. & 24 & $3 m$ & $96(88)$ \\
\hline 1n & & $2 a$ & $\mathrm{Bn}$ & Asc/ChCl & - & r.t. & 24 & $3 n$ & $96(88)$ \\
\hline 1a & $\mathrm{Ph}$ & $2 b$ & $\mathrm{Ph}$ & Asc/ChCl & - & r.t. & 24 & 30 & $91(85)$ \\
\hline 1a & $\mathrm{Ph}$ & $2 \mathrm{c}$ & & Asc/ChCl & - & r.t. & 24 & $3 p$ & $75(68)$ \\
\hline $1 \mathrm{a}$ & $\mathrm{Ph}$ & 2d & $\mathrm{CH}_{2} \mathrm{CH}_{2} \mathrm{OH}$ & Ass $/ \mathrm{ChCl}$ & - & r.t. & 24 & $3 q$ & $75(62)$ \\
\hline $1 \mathrm{a}$ & $\mathrm{Ph}$ & $2 \mathrm{e}$ & 4-MeO-Ph & Asc/ChCl & - & r.t. & 24 & $3 r$ & $90(84)$ \\
\hline
\end{tabular}

${ }^{a}$ Reactions were performed using $2 \mathrm{~g}$ of the corresponding solvent, $\mathrm{CuSO}_{4} \cdot 5 \mathrm{H}_{2} \mathrm{O} 10 \mathrm{Mol} \%$ and $1 \mathrm{mmol}$ of both reagents.

${ }^{b}$ Yields determined by ${ }^{1} \mathrm{H}$ NMR. ${ }^{c}$ Isolated yields.

The click-chemistry reactions in the investigated two green solvents were carried out using different azides containing aromatic or alkyl residues, 2a-e, and alkynes linked to a variety of functional groups, 1a-n. In particular, the acetylene moiety is linked to electron withdrawing or electron donating groups at the aromatic ring (1h-1k, Table 2), alkyl/cycloalkyl (1c, 1f and 1g, Table 2), cycloalkenyl (1e, Table 2), silane (1d, Table 2), alkyl/hydroxyl (1b, Table 2), amido (1l-1n, Table 2) and ether (1j and 11, Table 2) substituents. All the CuAAc reactions carried out in NADES GA/TMG, in the base-free experimental conditions, afforded for these reactions yields of triazole derivatives from excellent to quantitative, spanning from $78 \%$ to $99 \%$. The nature of the substituents and functional groups on the alkynes and azides have proved to not influence the course of the reaction carried out for 3 hours at $50^{\circ} \mathrm{C}$. Conversely, the CuAAc reactions carried out in active DES Asc/ChCl, in the base- and reducing agent-free reaction conditions, were strongly influenced by the nature of the substituents and functional groups on the alkynes. The solvent's ability to solubilize alkyl alkynes was the limiting factor of the process. In many cases the alkyne was completely immiscible with the solvent resulting in phase separation. This problem was particularly noticed with 1-pentyne 1c, cyclopropyl acetylene 1f, cyclohexenyl acetylene 1e and 3,3-dimethyl-1-butyne 1g (Table 2). However, these results agree with literature data which indicate that some DESs show good efficiency in extracting the aromatic compounds to the detriment of the aliphatic compounds because of their great affinity with aromatics. ${ }^{63}$ Overall, the click chemistry reactions in DES Asc/ChCl gave good results only with aromatic alkynes and the nature of the azides have shown to moderately influence the formation of the triazole derivatives (75-97\% yield). 


\section{DESs recycle}

The recycling of green solvents is an important issue in order to increase the sustainability of a chemical process. Thus, we investigated the recycling capabilities of the tested best green solvents in our set. Therefore, the CuAAc optimized reaction between phenyl acetylene and benzyl azide in the two NADES GA/TMG and Asc/ChCl was used as a model reaction. At the end of each experiment the formed products were extracted with ethyl acetate, a recommended solvent for its safety in use and for its low environmental and health impact. ${ }^{64}$

Table 3. Recycling study of the CuAAc reaction in natural deep eutectic solvents GA/TMG and Asc/ChCl. ${ }^{a}$

\begin{tabular}{lllll}
\hline Entry & Cycle & Solvent & Time $[\mathbf{h}]$ & Yield $^{b}$ \\
\hline 1 & 1 & GA/TMG & 3 & 99 \\
2 & 2 & GA/TMG & 3 & 99 \\
3 & 3 & GA/TMG & 3 & 90 \\
4 & 4 & GA/TMG & 3 & 68 \\
5 & 5 & GA/TMG & 3 & 10 \\
6 & 1 & Asc/ChCl & 24 & 97 \\
7 & 2 & Asc/ChCl & 24 & 85 \\
8 & 3 & Asc/ChCl & 24 & 30 \\
\hline
\end{tabular}

${ }^{a}$ Reactions were performed using $2 \mathrm{~g}$ of the corresponding solvent, $\mathrm{CuSO}_{4} \cdot 5 \mathrm{H}_{2} \mathrm{O} 10 \mathrm{Mol} \%$ and $1 \mathrm{mmol}$ of both reagents, for the GA/TMG solvent sodium ascorbate $30 \mathrm{Mol} \%$ was added. ${ }^{b}$ Yields determined by ${ }^{1} \mathrm{H}$ NMR.

In the case of GA/TMG, the initial reaction and the two subsequent recycling processes proceeded with good yields (entries 1-3, Table 3). A moderate loss of efficiency was found in the fourth cycle and a drastic loss of yield in the fifth cycle (entries 4 and 5, Table 3). The results are attributable to the degradation of sodium ascorbate, detectable by the browning of the reaction mixture and by the leaching catalyst during the extraction process. In case of Asc/ $\mathbf{C h C l}$, there is a loss of efficiency accompanied by a strong darkening of the solution already from the second cycle, that is due to the degradation of the L-ascorbic acid operated by air and light ${ }^{65}$ and photochemical oxidation ${ }^{66}$ (Table $3)$.

\section{Theoretical calculations}

The possibility of successfully carrying out the reaction in absence of triethylamine (a base commonly used for the development of the click reaction in order to facilitate the $\mathrm{C}-\mathrm{H}$ bond cleavage in the alkyne) ${ }^{67}$ was furtherly explored via DFT measures. These structural computational studies were 
carried out to investigate whether and how the active DESs can participate to the reaction via deprotonation of the alkyne or participation of the hydrogen in the H-bonds network of the liquids. The two liquids Asc/ ChCl and GA/TMG were analyzed in these studies.

The choline chloride/ L-ascorbic acid system (Asc/ChCl) has been modeled by DFT calculations in the presence and absence of phenylacetylene (P1) (PBE-D3/def2-TZVP level, see Computational Details).

In the absence of $\mathbf{P 1}$, the alcohol groups on both L-ascorbic acid and choline establish hydrogen bonds with the chloride (Figure 2), similarly to what urea does. ${ }^{68}$ The ammonium group of choline is electrostatically attracted by the electron-rich lactonic moiety of L-ascorbic acid.

In the presence of the alkyne, the structure modifies to accommodate $\mathbf{P 1}$ in the weak interaction network (Asc/ChCl/P1). The formation Gibbs energy of the adduct is negative with respect to the isolated moieties (-20.5 kcal/mol), ensuring its thermodynamical stability, and a complex network of weak interactions can be observed (Figure 2): the chloride interacts with three hydroxyl groups, one belonging to the choline and two to the L-ascorbic acid. P1 interacts with the chloride, too, through its terminal $\mathrm{CH}$. The ammonium group of choline is positioned close to the electron-rich part of $\mathbf{P 1}$ and one oxygen of L-ascorbic acid establishes a hydrogen bond with the ortho proton of P1. Weaker hydrogen bonds exist between the oxygens of the L-ascorbic acid and the $\mathrm{CH}$ of the ammonium moiety.

Noteworthy, the terminal C-H bond of P1 in the adduct is $1.083 \AA$, whereas for an isolated P1 the same bond would be $1.070 \AA$. This lengthening, with the consequent bond weakening, can likely explain the easier abstraction of the terminal proton.

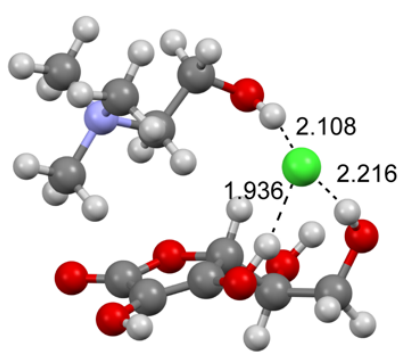

$\mathrm{Asc} / \mathrm{ChCl}$

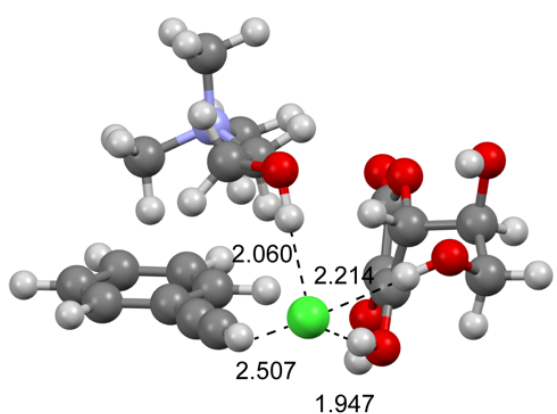

Asc/ChCl/P1

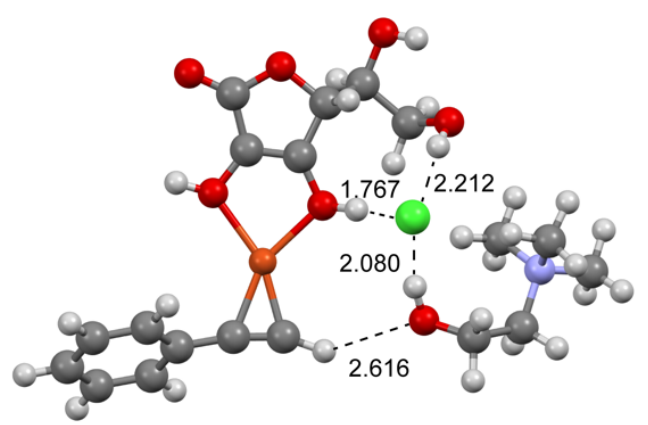

$\mathrm{Asc} / \mathrm{ChCl} / \mathrm{P} 1 / \mathrm{Cu}$

Figure 2. DFT-optimized geometries of the Asc/ChCl, Asc/ChCl/P1 and Asc/ChCl/P1/Cu adducts. Relevant distances are expressed in $\AA$. 
Upon the reduction of $\mathrm{Cu}(\mathrm{II})$ to $\mathrm{Cu}(\mathrm{I})$, the latter coordinates the triple bond and the L-ascorbic acid at the same time, stabilizing the complex $(\mathbf{A s c} / \mathbf{C h C l} / \mathbf{P 1} / \mathbf{C u})$. Simultaneously, the terminal proton interacts with the oxygen of the choline, again weakening the bond, whereas the chloride is surrounded by alcohol protons. After this intermediate, the reaction can proceed as usual, with proton abstraction, formation of the $\mathrm{C}-\mathrm{Cu} \sigma$ bond and reaction with the azide. ${ }^{69}$

A similar investigation was carried out for the GA/TMG DES (Figure 3). In the absence of P1, the alcohol group of one GA and the carboxylic group of the second GA interact with the carboxylate moiety of the trimethylglycine. At the same time, the two GA units interact each other with an additional, weak hydrogen bond. Introducing the P1 in the structure (GA/TMG/P1), the Gibbs energy is $-9.1 \mathrm{kcal} / \mathrm{mol}$. In the optimized geometry, the described arrangement does not change very much, but the terminal proton of $\mathbf{P 1}$ interacts with the available carboxylic group of the GA, causing an elongation just slightly smaller than before (1.79 $\AA$ ). Also, in this case, the solvent can act as a ligand, stabilizing the $\mathrm{Cu}(\mathrm{I})$-alkyne complex $(\mathbf{G A} / \mathbf{T M G} / \mathbf{P 1} / \mathbf{C u})$, and as a basic centre, favouring the proton cleavage and the $\sigma$-coordination of the copper.

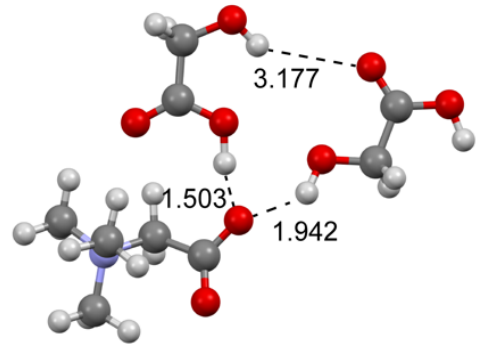

GA/TMG

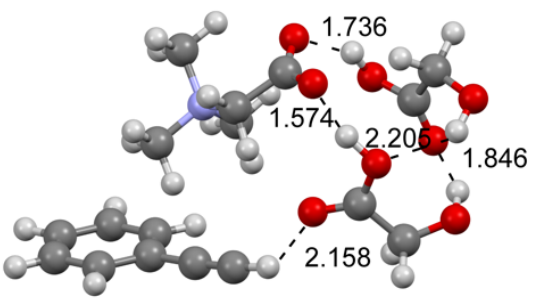

GA/TMG/P1

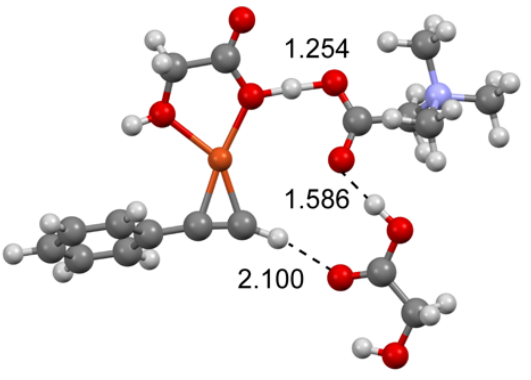

GA/TMG/P1/Cu

Figure 3. DFT-optimized geometries of the Asc/ChCl, Asc/ChCl/P1 and Asc/ChCl/P1/Cu adducts. Relevant distances are expressed in $\AA$.

Notably, also when the same reaction is carried out in standard polar solvents, as acetonitrile for example, the coordination of the solvent is generally invoked to stabilize the $\mathrm{Cu}(\mathrm{I})$-alkyne complex. ${ }^{70}$

\section{CONCLUSIONS}

In summary, in this work we reported the investigation of a set of natural deep eutectic solvents (NADESs) as "active" reaction media in the copper-catalysed azide-alkyne cycloaddition reactions (CuAAc). In all the cases, the reactions were carried out in the absence of a base, did not need high temperatures, tolerated several functional groups, did not require an external reducing agent (in the 
cases of the use of the reducing DESs) and allowed the recycling of the solvent. As reported, the DES GA/TMG represented the best green solvent in our set to obtain 1,2,3-triazoles substituted with different functional groups in good yield. Asc/ChCl DES showed also many advantages because its reducing capabilities avoided the use of any reducing agent in the reaction mixture. In addition, the use of the green solvent Asc/ $\mathbf{C h C l}$ as active DES in a non-classical CuAAc base- and reducing agentfree reaction contribute to the use of active green solvents in catalytic processes with advantages in terms of atom economy. DFT studies showed that the $\mathrm{C}-\mathrm{H}$ alkyne bond enters in the H-bonds network of the DESs, being elongated and giving a rationale for the absence of a catalytic base, and that the NADES constituents are able to stabilize the copper intermediates. To the best of our knowledge, this is the first example of this kind of reaction mechanism operated by these active green solvents and it could be relevant for other base-catalysed reaction procedures. These reaction strengths outline the great potential of NADES for CuAAc and it can contribute to the elimination of common solvents in organic synthesis and in catalysis.

\section{CONFLICTS OF INTEREST}

There are no conflicts to declare.

\section{EXPERIMENTAL}

\section{General Information}

Solvents and reagents were used as received from commercial sources. The experiments were performed in a glass sealed tube with a magnetic stirrer. NMR spectra $\left({ }^{1} \mathrm{H}-\mathrm{NMR}\right.$ recorded at 500 $\mathrm{MHz},{ }^{13} \mathrm{C}$-NMR recorded at $125 \mathrm{MHz}$ ) were obtained in $\mathrm{CDCl}_{3}$ solution on a Varian instrument (Agilent Technologies, Palo Alto, CA, USA), and data are reported in ppm relative to TMS as an internal standard. Benzyl/alkyl and aromatic azides were synthesized according to literature procedures. ${ }^{71}$ Isolated yield was determined after addition of water to the crude reaction mixture and filtration of the desired triazole or after extraction with ethyl acetate and purification by flash chromatography when desired triazole is an oil.

\section{Deep Eutectic Solvents Preparation}

The DESs were prepared following the procedures reported in previous papers: ${ }^{.2}$ the solid components were weighted in a flask at the proper molar ratio and then were mixed and heated at $50^{\circ} \mathrm{C}$ until a homogeneous liquid was formed in times spanning from 30 mins to 2 hours. 
General procedure for the synthesis of 1,2,3-triazole derivatives 3a-r in deep eutectic solvent GA/TMG: synthesis of 1-benzyl-4-phenyl-1H-1,2,3-triazole (3a). In a glass sealed tube benzyl azide $(0.133 \mathrm{~g}, 1 \mathrm{mmol})$, phenylacetylene $(0.112 \mathrm{~mL}, 1 \mathrm{mmol})$, copper sulphate $(0.016 \mathrm{~g}, 10 \mathrm{~mol} \%)$ and sodium ascorbate $(59.4 \mathrm{mg}, 30 \mathrm{~mol} \%)$ in $2 \mathrm{~mL}$ of green solvent were added, then it was putted in a oil bath heated at $50{ }^{\circ} \mathrm{C}$. The mixture was stirred for 3 hours. Addition of water $(10 \mathrm{~mL})$ to the crude reaction mixture allows the easy separation by filtration of the desired product $\mathbf{3 a}$ with $96 \%$ yield as a white powder $(0.226 \mathrm{~g})$.

General procedure for the synthesis of 1,2,3-triazole derivatives 3a-r in deep eutectic solvent Asc/ChCl: synthesis of 1-benzyl-4-phenyl-1H-1,2,3-triazole (3a). In a glass sealed tube benzyl azide $(0.133 \mathrm{~g}, 1 \mathrm{mmol})$, phenylacetylene $(0.112 \mathrm{~mL}, 1 \mathrm{mmol})$ and copper sulphate $(0.016 \mathrm{~g}, 10$ $\mathrm{mol} \%$ ) in $2 \mathrm{~mL}$ of green solvent were added. The mixture was stirred for 24 hours at room temperature. and was extracted with ethyl acetate $(3 \times 3 \mathrm{~mL})$. Addition of water $(10 \mathrm{~mL})$ to the crude reaction mixture allows the easy separation by filtration of the desired product $\mathbf{3 a}$ with $94 \%$ yield as a white powder $(0.221 \mathrm{~g})$.

General procedure for the recycling green solvents. The CuAAc optimized reaction between phenyl acetylene and benzyl azide in the two natural deep eutectic solvent GA/TMG and Asc/ChCl was used as a model reaction. After each extraction cycle with ethyl acetate, azide and alkyne were added again to the catalyst system and the reaction mixture was stirred for the required time. This operation was repeated several times (see Table 3).

Computational details: All the structures have been optimized by ORCA $4.1 .0^{73}$ using the PBE functional in conjunction with a def2-TZVP basis set (grid $=5$ ). The dispersion forces have been taken into account by using the Grimme's correction D3 with BJ damping. ${ }^{74,75}$ The thermodynamic properties have been computed at the same level of theory, showing that all the adducts are true minima of the potential energy surface (no negative vibrational frequencies).

\section{REFERENCES}

1 Y. Zhu, J. Xie, F. Huang and L. Cao, Science of The Total Environment, 2020, 727, 138704.

2 D. Fattorini and F. Regoli, Environmental Pollution, 2020, 264, 114732.

3 D. A. Glencross, T.-R. Ho, N. Camiña, C. M. Hawrylowicz and P. E. Pfeffer, Free Radical Biology and Medicine, 2020, 151, 56-68. 

Engineering Journal, 2019, 372, 992-1006.

$7 \quad$ Ionic Liquids in Synthesis, Wiley, 2007.

R. D. Rogers, Science, 2003, 302, 792-793.

L. Brinchi, R. Germani, E. Braccalenti, N. Spreti, M. Tiecco and G. Savelli, Journal of Colloid and Interface Science, 2010, 348, 137-145.

D. Zhao, Y. Liao and Z. Zhang, CLEAN - Soil, Air, Water, 2007, 35, 42-48.

D. Coleman and N. Gathergood, Chemical Society Reviews, 2010, 39, 600.

H.-G. Liao, Y.-X. Jiang, Z.-Y. Zhou, S.-P. Chen and S.-G. Sun, Angewandte Chemie International Edition, 2008, 47, 9100-9103.

L. L. Sze, S. Pandey, S. Ravula, S. Pandey, H. Zhao, G. A. Baker and S. N. Baker, ACS Sustainable Chemistry \& Engineering, 2014, 2, 2117-2123.

A. Abo-Hamad, M. Hayyan, M. A. AlSaadi and M. A. Hashim, Chemical Engineering Journal, 2015, 273, 551-567.

D. A. Alonso, A. Baeza, R. Chinchilla, G. Guillena, I. M. Pastor and D. J. Ramón, European Journal of Organic Chemistry, 2016, 2016, 612-632.

S. C. Cunha and J. O. Fernandes, TrAC Trends in Analytical Chemistry, 2018, 105, 225-239.

D. O. Abranches, M. A. R. Martins, L. P. Silva, N. Schaeffer, S. P. Pinho and J. A. P. Coutinho, Chemical Communications, 2019, 55, 10253-10256.

D. J. G. P. van Osch, C. H. J. T. Dietz, S. E. E. Warrag and M. C. Kroon, ACS Sustainable Chemistry \& Engineering, 2020, acssuschemeng.0c00559.

R. Germani, M. Orlandini, M. Tiecco and T. del Giacco, Journal of Molecular Liquids, 2017, 240, 233239.

D. O. Abranches, L. P. Silva, M. A. R. Martins, S. P. Pinho and J. A. P. Coutinho, ChemSusChem, 2020, 13, 4916-4921.

C. F. Araujo, J. A. P. Coutinho, M. M. Nolasco, S. F. Parker, P. J. A. Ribeiro-Claro, S. Rudić, B. I. G. Soares and P. D. Vaz, Physical Chemistry Chemical Physics, 2017, 19, 17998-18009.

F. Gabriele, M. Chiarini, R. Germani, M. Tiecco and N. Spreti, Journal of Molecular Liquids, 2019, 291, 111301.

D. v. Wagle, G. A. Baker and E. Mamontov, The Journal of Physical Chemistry Letters, 2015, 6, 29242928.

M. Hayyan, M. A. Hashim, A. Hayyan, M. A. Al-Saadi, I. M. AlNashef, M. E. S. Mirghani and O. K. Saheed, Chemosphere, 2013, 90, 2193-2195. 

Engineering, 2015, 3, 3398-3404.

27 Y. Liu, J. B. Friesen, J. B. McAlpine, D. C. Lankin, S.-N. Chen and G. F. Pauli, Journal of Natural Products, 2018, 81, 679-690.

Y. Dai, J. van Spronsen, G.-J. Witkamp, R. Verpoorte and Y. H. Choi, Analytica Chimica Acta, 2013, 766, 61-68.

A. Gertrudes, R. Craveiro, Z. Eltayari, R. L. Reis, A. Paiva and A. R. C. Duarte, ACS Sustainable Chemistry \& Engineering, 2017, 5, 9542-9553.

R. Craveiro, F. Mano, A. Paiva and A. R. C. Duarte, in Deep Eutectic Solvents, 2019, pp. 95-110.

Y. H. Choi, J. van Spronsen, Y. Dai, M. Verberne, F. Hollmann, I. W. C. E. Arends, G.-J. Witkamp and R. Verpoorte, Plant Physiology, 2011, 156, 1701-1705. Gonzalez, Journal of Molecular Liquids, 2019, 293, 111452.

M. Tiecco, D. A. Alonso, D. R. Ñíguez, G. Ciancaleoni, G. Guillena, D. J. Ramón, A. A. Bonillo and R. Germani, Journal of Molecular Liquids, 2020, 313, 113573.

T. Palomba, G. Ciancaleoni, T. del Giacco, R. Germani, F. Ianni and M. Tiecco, Journal of Molecular Liquids, 2018, 262, 285-294.

A. di Crescenzo, M. Tiecco, R. Zappacosta, S. Boncompagni, P. di Profio, V. Ettorre, A. Fontana, R. Germani and G. Siani, Journal of Molecular Liquids, 2018, 268, 371-375.

M. Ballarotto, F. Cappellini, R. Maestri, T. del Giacco, P. di Profio, M. Tiecco and R. Germani, Monatshefte für Chemie - Chemical Monthly, 2020, 151, 1387-1394.

P. Liu, J.-W. Hao, L.-P. Mo and Z.-H. Zhang, RSC Advances, 2015, 5, 48675-48704.

S. Nejrotti, M. Iannicelli, S. S. Jamil, D. Arnodo, M. Blangetti and C. Prandi, Green Chemistry, 2020, 22, 110-117.

S. K. Shukla, D. Nikjoo and J.-P. Mikkola, Physical Chemistry Chemical Physics, 2020, 22, 966-970.

T. Palomba, G. Ciancaleoni, T. del Giacco, R. Germani, F. Ianni and M. Tiecco, Journal of Molecular Liquids, 2018, 262, 285-294.

M. Tiecco, D. A. Alonso, D. R. Ñíguez, G. Ciancaleoni, G. Guillena, D. J. Ramón, A. A. Bonillo and R. Germani, Journal of Molecular Liquids, 2020, 313, 113573.

V. v. Rostovtsev, L. G. Green, V. v. Fokin and K. B. Sharpless, Angewandte Chemie International Edition, 2002, 41, 2596-2599.

H. C. Kolb, M. G. Finn and K. B. Sharpless, Angewandte Chemie International Edition, 2001, 40, 2004-2021.

B. R. Buckley, S. E. Dann, H. Heaney and E. C. Stubbs, European Journal of Organic Chemistry, 2011, 2011, 770-776. 
47 X. Jiang, X. Hao, L. Jing, G. Wu, D. Kang, X. Liu and P. Zhan, Expert Opinion on Drug Discovery, 2019, 14, 779-789.

M. Arslan and M. A. Tasdelen, Chemistry Africa, 2019, 2, 195-214.

D. Iannazzo, A. Pistone, A. Visco, G. Galtieri, S. v Giofrè, R. Romeo, G. Romeo, S. Cappello, M. Bonsignore, R. Denaro and S. Galvagno, Materials Research Express, 2015, 2, 115001.

D. Iannazzo, A. Pistone, I. Ziccarelli, C. Espro, S. Galvagno, S. v Giofré, R. Romeo, N. Cicero, G. D. Bua, G. Lanza, L. Legnani and M. A. Chiacchio, Environmental Science and Pollution Research, 2017, 24, 14735-14747.

A. P. Abbott, G. Capper, D. L. Davies, R. K. Rasheed and V. Tambyrajah, Chemical Communications, 2003, 70-71.

J. M. Silva, R. L. Reis, A. Paiva and A. R. C. Duarte, ACS Sustainable Chemistry \& Engineering, 2018, 6, 10355-10363.

U. N. Yadav and G. S. Shankarling, Journal of Molecular Liquids, 2014, 191, 137-141.

A. Mocan, A. Diuzheva, S. Bădărău, C. Moldovan, V. Andruch, S. Carradori, C. Campestre, A. Tartaglia, M. de Simone, D. Vodnar, M. Tiecco, R. Germani, G. Crișan and M. Locatelli, Molecules, 2019, 24, 1226.

F. Cardellini, M. Tiecco, R. Germani, G. Cardinali, L. Corte, L. Roscini and N. Spreti, RSC Adv., 2014, 4, 55990-56002.

C. Vidal and J. García-Álvarez, Green Chemistry, 2014, 16, 3515.

W. Riemenschneider and M. Tanifuji, in Ullmann's Encyclopedia of Industrial Chemistry, 2011.

R. Huisgen, 1,3-Dipolar Cycloaddition Chemistry, Wiley, New York, 1984.

K. v. Krishnamurty and G. M. Harris, Chemical Reviews, 1961, 61, 213-246.

M. Lim and M.-J. Kim, Chemosphere, 2013, 90, 1526-1532.

N. R. Rodriguez, P. F. Requejo and M. C. Kroon, Industrial \& Engineering Chemistry Research, 2015, 54, 11404-11412.

D. Prat, A. Wells, J. Hayler, H. Sneddon, C. R. McElroy, S. Abou-Shehada and P. J. Dunn, Green Chemistry, , DOI:10.1039/C5GC01008J.

S. M. Blaug and B. Hajratwala, Journal of Pharmaceutical Sciences, 1972, 61, 556-562.

I. Ahmad, M. A. Sheraz, S. Ahmed, R. H. Shaikh, F. H. M. Vaid, S. ur Rehman Khattak and S. A. Ansari, AAPS PharmSciTech, 2011, 12, 917-923.

M. Meldal and C. W. Tornøe, Chemical Reviews, 2008, 108, 2952-3015.

A. P. Abbott, G. Capper, D. L. Davies, R. K. Rasheed and V. Tambyrajah, Chemical Communications, 2003, 70-71. 
70 F. Himo, T. Lovell, R. Hilgraf, V. v. Rostovtsev, L. Noodleman, K. B. Sharpless and V. v. Fokin, Journal of the American Chemical Society, 2005, 127, 210-216.

71 S. Giofrè, R. Romeo, C. Carnovale, R. Mancuso, S. Cirmi, M. Navarra, A. Garozzo and M. Chiacchio, Molecules, 2015, 20, 5260-5275.

72 F. Cardellini, R. Germani, G. Cardinali, L. Corte, L. Roscini, N. Spreti and M. Tiecco, RSC Advances, 2015, 5, 31772-31786.

73 F. Neese, WIREs Computational Molecular Science, 2018, 8, e1327.

74 S. Grimme, S. Ehrlich and L. Goerigk, Journal of Computational Chemistry, 2011, 32, 1456-1465.

75 S. Grimme, J. Antony, S. Ehrlich and H. Krieg, The Journal of Chemical Physics, 2010, 132, 154104. 